\title{
GMR
}

\section{Comparative analysis of MIR168 promoters in three plant species}

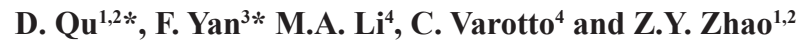

${ }^{1}$ College of Horticulture, Northwest A\&F University, Yangling, Shaanxi, China

${ }^{2}$ Apple E\&T Research Centre of Shaanxi Province, Yangling, Shaanxi, China ${ }^{3}$ Shaanxi Province Key Laboratory of Bio-resources, Shaanxi University of Technology, Hanzhong, China

${ }^{4}$ Department of Biodiversity and Molecular Ecology, Fondazione Edmund Mach, Michele all'Adige, TN, Italy

*These authors contributed equally to this study.

Corresponding author: Z.Y. Zhao

E-mail: zhaozy@nwsuaf.edu.cn / applebreed@126.com

Genet. Mol. Res. 15 (2): gmr.15027684

Received September 18, 2015

Accepted December 10, 2015

Published May 25, 2016

DOI http://dx.doi.org/10.4238/gmr.15027684

\begin{abstract}
MicroRNAs (miRNAs) play important roles in the regulation of gene expression by post-transcriptionally targeting mRNAs for cleavage or translational repression. miR168 is a key miRNA because it regulates the expression of the slicer protein ARGONAUTE1 (AGO1), which catalyzes mRNA cleavage. Interestingly, plant miR168s are highly evolutionarily conserved; however, it is unclear whether MIR168 promoter elements and expression patterns are also conserved. Here, we isolated MIR168 promoters from monocot rice and dicot grape genomes. To determine the expression pattern, different promoters were fused to a beta-glucoronidase reporter gene and the resulting constructs were then transformed in Arabidopsis. The results revealed clear differences in the MIR168 promoter sequence of monocot and dicot plant species. Moreover, the pattern of MIR168 promoter expression differed between monocots and dicots. These
\end{abstract}


results suggest that, unlike that of miR168, the MIR168 promoter is not conserved in monocots and dicots.

Key words: MIR168; Promoter; Rice; Grape

\section{INTRODUCTION}

MicroRNAs (miRNAs) are a large class of short non-coding RNAs (19-25 nt long), which have emerged as important post-transcriptional regulators of protein-coding genes and target mRNAs for cleavage or translational repression (Sunkar and Zhu, 2004; Bartel, 2004; Voinnet, 2009). miRNAs have important regulatory roles in multiple fundamental processes in plant growth and development, and in the regulation of plant gene silencing (Sunkar and Zhu, 2004; Mallory and Vaucheret, 2006). Moreover, miRNAs are involved in various responses to biotic and abiotic stresses, such as oxidative, drought, mineral nutrient deficiency, and fungal invasion stress (Navarro et al., 2006; Lu and Huang, 2008; Khraiwesh et al., 2012; Gupta et al., 2014).

Among the miRNAs, miR168 represents a group of plant-specific miRNAs, whose target gene, ARGONAUTE1 protein (AGO1), is the basic component of RNA-induced silencing complex (RISC), which is involves in the cleavage of target mRNAs. miR168 plays a pivotal role as it regulates the expression of AGO1, a mechanism through which all microRNAs can down-regulate their targets (Vaucheret et al., 2006; Gazzani et al., 2009). In addition, miR168 is reportedly involved in the regulation of plant growth, fruit development, and response to different stresses, such as UV-B and aluminum, and is also up-regulated during viral infection in plants, during which it is ubiquitous in plant-virus interactions (Várallyay et al., 2010; Lima et al., 2011; Wang et al., 2013; Xian et al., 2014).

Plant miRNAs are evolutionarily conserved in a wide variety of species, ranging from mosses and ferns to dicots (Arazi et al., 2005; Jones-Rhoades et al., 2006; Fattash et al., 2007). Interesting, miR 168a are also highly conserved in different plants, from rice to Arabidopsis (Table 1). Despite an increasing number of reports predicting miRNAs and demonstrating their roles in the development and morphogenesis of plants, relatively little is known about the conservation of miR168 regulatory elements in various plant species (Han et al., 2014). It is generally accepted that the ACGT core element (ACE) is functionally important in a variety of promoters that respond to different stresses, such as light (Donald and Cashmore, 1990), jasmonic acid (Mason et al., 1993), and hormones stresses (Shen et al., 1993). The ACGT core sequences are found in Arabidopsis thaliana and also in rice (Mundy et al., 1990), wheat (Marcotte et al., 1989), and barley (Shen et al., 1996), suggesting that ACEs are conserved across plant species. Moreover, the copy number of ACGT elements in a promoter can significantly affect gene expression (Mehrotra et al., 2005). In this study, we compared regulatory differences (transcription factor binding sites and expression patterns) controlling the expression of miR168 in different plant families. These findings could lead to a better understanding of miR168 biogenesis and regulatory mechanisms for use in research in different plant species, from monocots to dicots.

\section{MATERIAL AND METHODS}

\section{Plant material}

Leaves of Oryza sativa were collected in Trentino Alto Adige (Italy). Tissue was 
collected from Vitis vinifera, which were grown in a greenhouse from leaves collected in Trentino Alto Adige (Italy). A. thaliana, ecotype Columbia, was used as the wild-type control in this study and grown under long photoperiod conditions $\left(16 \mathrm{~h} \mathrm{light} / 8 \mathrm{~h}\right.$ dark) at $25^{\circ} \mathrm{C}$ in a greenhouse. Transgenic Arabidopsis seeds were surface sterilized in 5\% hypochlorite solution, treated with $70 \%$ ethanol, and rinsed in distilled water. Sterilized seeds were plated on solidified Murashige-Skoog (MS) medium with kanamycin, supplemented with 1\% sucrose. T1 seeds were germinated and grown in a controlled chamber at $20^{\circ} \mathrm{C}$ and $40 \%$ relative humidity under continuous illumination.

Table 1. MIR168 sequences of different plant species.

\begin{tabular}{l|l}
\hline Species & MIR168 sequence \\
\hline OrySat & Ucgcuuggugcagaucgggac (osa-MIR168a) \\
\hline VitVin & Ucgcuuggugcaggucgggaa (vvi-MIR168) \\
\hline PopTri & Ucgcuuggugcaggucgggaa (ptc-MIR168a) \\
\hline SolLyc & Ucgcuuggugcaggucgggac (sly-MIR168a) \\
\hline AraTha & Ucgcuuggugcaggucgggaa (ath-MIR168a) \\
\hline
\end{tabular}

\section{Isolation of MIR168 promoter in different species}

Genomic DNA of $O$. sativa and $V$. vinifera was extracted from leaves using the Hexadecyltrimethy Ammonium Bromide (CTAB) method (Doyle, 1987). The upstream fragments of MIR168 were amplified from genomic DNA using the primers detailed in Table 2. Primers were designed on conserved upstream sequences of MIR168 or on the highly conserved sequences of the mature miR168 and miR168*. Amplification products were cloned in pGEM-T (Promega) vectors. At least eight clones corresponding to each product were sequenced bi-directionally to confirm their identity. Multiple sequence alignments were performed with manual editing in Bioedit. PLANTCARE was used to analyze promoter core elements (http://bioinformatics.psb.ugent.be/webtools/plantcare/html/).

Table 2. PCR primer sequences.

\begin{tabular}{l|l}
\hline Species & Primers \\
\hline OrySat & F: 5'-CACCTTTGAAACGAGGGATTTTTATAGG-3' \\
& R: 5'-CGGTACCGGCGCCAAGAGACGAGACGAGACG-3' \\
\hline VitVin & F: 5'-GACACTTCCTAGCATCTTCAATACA-3' \\
& R: 5'-CGATTCAGTTGATGCAAGGCGGGA-3' \\
\hline
\end{tabular}

\section{Expression analysis of pMIR168 from different species}

The determined $p M I R 168$ regions from three species were cloned into the Gateway $\mathrm{pENTR/D-TOPO}$ vector (Life Technologies) according to the manufacturer instructions. The resulting plasmids were recombined with PKGWFS7 to drive the expression of an enhanced green fluorescent protein-beta glucuronidase (eGFP-uidA) fusion reporter construct. The three constructs were pOrySatMIR168a:GUS, pVitVinMIR168:GUS, and pAraThaMIR168a: GUS. Each plasmid was used to transform 4-week-old A. thaliana plants by the floral dipping method. A total of 50-60 primary transgenic plants were screened per construct by histochemical $\beta$-Glucuronidase (GUS) staining. 


\section{Histochemical GUS staining}

T1 7-day-old seedlings were harvested. Histochemical GUS staining was performed following the standard procedure (Jefferson et al., 1987) with a modified buffer containing $1 \mathrm{mg} / \mathrm{mL}$ X-GLUC (5-bromo-4-chloro-3-indolyl- $\beta$-D-glucuronic cyclohexylamine salt), 50 $\mathrm{mM}$ sodium phosphate ( $\mathrm{pH} 7.0$ ), $2 \mathrm{mM}$ potassium ferricyanide, $2 \mathrm{mM}$ potassium ferrocyanide, and $10 \mathrm{mM}$ Ethylenediaminetetraaceticacid (EDTA). Plant seedlings were incubated in buffer overnight at $37^{\circ} \mathrm{C}$ in the dark. Prior to observation, tissue samples were cleared with a graded ethanol dehydration series to $70 \%$ ethanol. For GUS quantification, at least 50 independent transgenic lines for each construct were assessed. At least 12 repeats were carried out per line. Microscopic inspection was carried out on a Leica micrscope (Leica MZ16F).

\section{RESULTS AND DISSCUSSION}

\section{DNA sequence variation in MIR168 promoter regions}

miR168 sequences are conserved between monocotyledonous and dicotyledonous plants (Table 1). However, the most interesting results relate to the copy number of the G-box (CACGTG) of MIR168 promoters from different species were different. G-box (CACGTG) is the most prevalent G-box in all ACGT classes ACGT classes (Figure 1 and $\underline{\text { S1 Figure) }}$ (Mehrotra et al., 2013). The MIR168 promoter of A. thaliana contains three G-box motifs (CACGTG) (Gazzani et al., 2009). However, no G-box motif was found in the MIR168 promoter of monocot species like rice, and two were found in the MIR168 promoter of dicot species such as grape. Therefore, the evolution of the MIR168 promoter sequence does not correspond closely with that of MIR168 sequences. It is reported that these G-boxes are differentially regulated by the distance between two copies of the motif (Mehrotra and Mehrotra, 2010). Moreover, the G-box copy number in a promoter, and the distance from the transcriptional start site, also result in drastically altered gene expression, causing changes in the pattern of expression and even in resistance (Mehrotra et al., 2005, 2013). Thus, the degree of conservation of different regulatory elements may decrease depending on the motif in monocots and dicots. Of note, no G-box motif was present in the promoter of monocot species, such as rice. Given the variable copy number of the ACGT core sequence, attempts should be made to analyze the expression patterns of the ACGT core element in MIR168 promoters of different plant species.

\section{Differential expression of MIR168 promoters in transgenic plants}

To further investigate whether differences in the MIR168 promoters of monocots and dicots determined the similarities and differences in expression, promoters from different species were transformed in A. thaliana. We generated a reporter line in which the beta-GUS coding region was placed under the control of MIR168 promoter fragments (pOrySatMIR168a:GUS, pVitVinMIR168:GUS). After GUS staining was performed on single copy transgenic lines, the results showed there were obvious differences in expression patterns among angiosperm, suggesting that the expression patterns of miR168 genes are not conserved (Figure 2, 3, and 4). Two patterns can be observed in pOrySatMIR168a: GUS transgenic plants (Figure 3 ), and GUS staining can only be detected in the blade tip (Figure 3A) and emerging leaf 
primordia (Figure 3B). However, more plant organs of GUS staining can be observed in the seedlings of pVitVinMIR168: GUS and pAraThaMIR168a: GUS transgenic plants, compared with those of pOrySatMIR168a: GUS transgenic plants (Figure 2 and 4). pVitVinMIR168: GUS and pAraThaMIR168a: GUS transgenic plants displayed the same pattern of staining as pOrySatMIR168a: GUS plants. Moreover, the vasculature of the cotyledons was also stained, and GUS expression was observed in the lateral and primary root tips of pVitVinMIR168: GUS transformed plants (Figure 4).
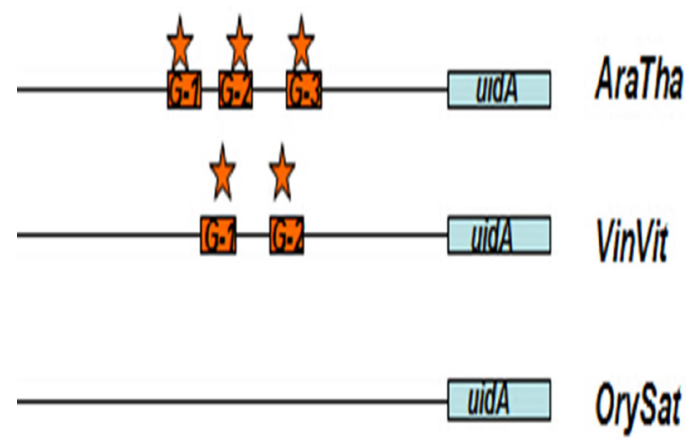

Figure 1. pAraThaMIR168a, pVitVinMIR168, and pOrySatMIR168a:GUS constructs are represented. G-box copy numbers in MIR168 promoters of Arabidopsis, grape, and rice are shown. Orange box: G-box.

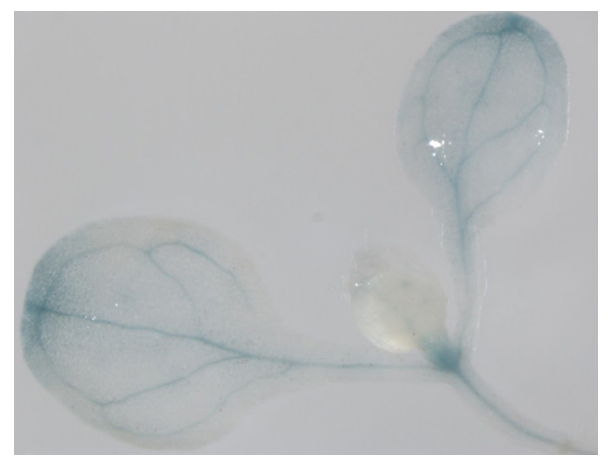

Figure 2. GUS-staining of transformed Arabidopsis lines carrying the pAraThaMIR168a:GUS constructs. GUS staining can be observed in the blade tip, vasculature of the cotyledons, and emerging leaf primodia.

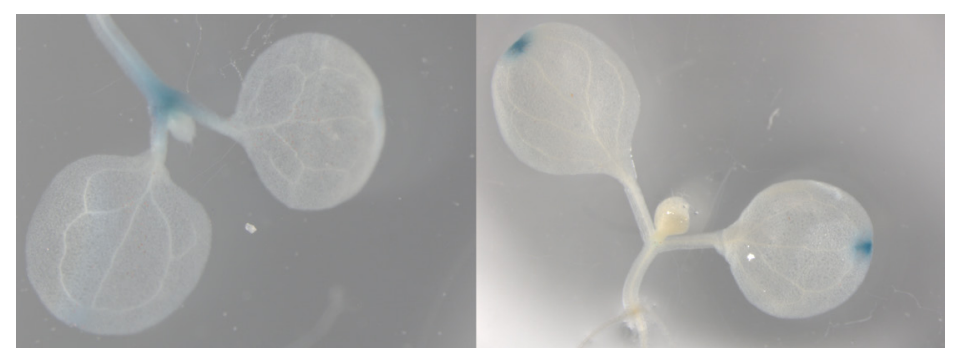

Figure 3. Histochemical analysis of pOrySatMIR168a:GUS overexpression. A. and B. Two patterns of GUS expression. GUS staining can be observed in the emerging leaf primodia and blade tip. 


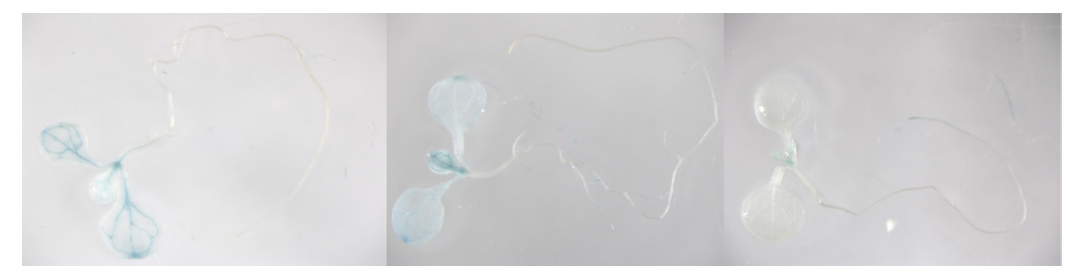

Figure 4. Histochemical analysis of pVitVinMIR168:GUS overexpression. Three patterns were observed. A. GUS expression in emerging leaf, primodia vasculature of the cotyledons and the blade tip. B. GUS staining in the lateral root tip, and C shows GUS staining in the primary root tip.

The relative position of the ACGT core motif, and its copy number upstream of the promoter, affects the recognition of transcription factors and therefore, affects development and the response to different environments, such as flower development and pathogen defense (Chuang et al., 1999; Zhou et al., 2000; Mehrotra et al., 2005). Multiple ACGT motifs, which bind synergistically to transcription factors, can form enhancer elements (Mehrotra et al., 2013). Our data suggest that there are no G-box motifs in the rice MIR168 promoter, whereas two and three G-boxes can be found in the grape and Arabidopsis MIR168 promoters, respectively. Additionally, there were significant differences in the expression patterns of G-boxes as controlled by promoters. We suggest that the apparent preference for a particular copy number shows that the MIR168 promoter of different species might be implicated in different physiological functions across monocots and dicots. In addition, variations in the correlation patterns between monocots and dicots might be implicated in the functional difference in transcriptional regulation. Therefore, further investigation of functional analysis would be of interest.

In conclusion, this is the first study that has attempted to analyze MIR168 promoters in monocots and dicots. Our results demonstrate that obvious differences exist within the MIR168 promoter sequences, although MIR168 sequences are highly conserved in monocots and dicots. We further show that the pattern of miR168 expression in different plant species differed in transformed plants due to the regulation of their MIR168 promoters. Therefore, it may be suggested that MIR168 promoters are not conserved between monocots and dicots. Further studies are required to clarify the regulatory relevance of these MIR168 promoters in monocot and dicots.

\section{Conflicts of interest}

The authors declare no conflict of interest.

\section{ACKNOWLEDGMENTS}

We thank Dr. Rui Meng for critically reading the manuscript. This work was supported by Modern Agro-industry Technology Research System (\#CARS-28).

\section{REFERENCES}

Arazi T, Talmor-Neiman M, Stav R, Riese M, et al. (2005). Cloning and characterization of micro-RNAs from moss. Plant J. 43: 837-848.http://dx.doi.org/10.1111/j.1365-313X.2005.02499.x

Bartel DP (2004). MicroRNAs: Genomics, biogenesis, mechanism, and function. Cell 116: 281-297.

Chuang CF, Running MP, Williams RW and Meyerowitz EM (1999). The PERIANTHIA gene encodes a bZIP protein involved in the determination of floral organ number in Arabidopsis thaliana. Genes Dev. 13: 334-344. http://dx.doi. org/10.1101/gad.13.3.334 
Donald RG and Cashmore AR (1990). Mutation of either G box or I box sequences profoundly affects expression from the Arabidopsis rbcS-1A promoter. EMBO J. 9: 1717-1726.

Doyle J (1987). A rapid DNA isolation procedure for small quantities of fresh leaf tissue. Phytochem. Bull. 19: 11-15.

Fattash I, Voss B, Reski R, Hess WR, et al. (2007). Evidence for the rapid expansion of microRNA-mediated regulation in early land plant evolution. BMC Plant Biol. 7: 13. http://dx.doi.org/10.1186/1471-2229-7-13

Gazzani S, Li M, Maistri S, Scarponi E, et al. (2009). Evolution of MIR168 paralogs in Brassicaceae. BMC Evol. Biol. 9: 1-14.http://dx.doi.org/10.1186/1471-2148-9-62

Gupta OP, Sharma P, Gupta RK and Sharma I (2014). Current status on role of miRNAs during plant-fungus interaction. Physiol. Mol. Plant Pathol. 85: 1-7. http://dx.doi.org/10.1016/j.pmpp.2013.10.002

Han J, Xie H, Kong ML, Sun QP, et al. (2014). Computational identification of miRNAs and their targets in Phaseolus vulgaris. Genet. Mol. Res. 13: 310-322.http://dx.doi.org/10.4238/2014.January.17.16

Jefferson RA, Kavanagh TA and Bevan MW (1987). GUS fusions: beta-glucuronidase as a sensitive and versatile gene fusion marker in higher plants. EMBO J. 6: 3901-3907.

Jones-Rhoades MW, Bartel DP and Bartel B (2006). MicroRNAS and their regulatory roles in plants. Annu. Rev. Plant Biol. 57: 19-53. http://dx.doi.org/10.1146/annurev.arplant.57.032905.105218

Khraiwesh B, Zhu JK and Zhu J (2012). Role of miRNAs and siRNAs in biotic and abiotic stress responses of plants. Biochim. Biophys. Acta 1819: 137-148. http://dx.doi.org/10.1016/j.bbagrm.2011.05.001

Lima JC, Arenhart RA, Margis-Pinheiro M and Margis R (2011). Aluminum triggers broad changes in microRNA expression in rice roots. Genet. Mol. Res. 10: 2817-2832. http://dx.doi.org/10.4238/2011.November.10.4

Lu XY and Huang XL (2008). Plant miRNAs and abiotic stress responses. Biochem. Biophys. Res. Commun. 368: 458462. http://dx.doi.org/10.1016/j.bbrc.2008.02.007

Mallory AC and Vaucheret H (2006). Functions of microRNAs and related small RNAs in plants. Nat. Genet. 38 (Suppl): S31-S36. http://dx.doi.org/10.1038/ng1791

Marcotte WR, Jr., Russell SH and Quatrano RS (1989). Abscisic acid-responsive sequences from the em gene of wheat. Plant Cell 1: 969-976. http://dx.doi.org/10.1105/tpc.1.10.969

Mason HS, DeWald DB and Mullet JE (1993). Identification of a methyl jasmonate-responsive domain in the soybean vspB promoter. Plant Cell 5: 241-251.

Mehrotra R and Mehrotra S (2010). Promoter activation by ACGT in response to salicylic and abscisic acids is differentially regulated by the spacing between two copies of the motif. J. Plant Physiol. 167: 1214-1218. http:// dx.doi.org/10.1016/j.jplph.2010.04.005

Mehrotra R, Kiran K, Chaturvedi CP, Ansari SA, et al. (2005). Effect of copy number and spacing of the ACGT and GT cis elements on transient expression of minimal promoter in plants. J. Genet. 84: 183-187. http://dx.doi.org/10.1007/ BF02715844

Mehrotra R, Sethi S, Zutshi I, Bhalothia P, et al. (2013). Patterns and evolution of ACGT repeat cis-element landscape across four plant genomes. BMC Genomics 14: 1735-1742.http://dx.doi.org/10.1186/1471-2164-14-203

Mundy J, Yamaguchi-Shinozaki K and Chua NH (1990). Nuclear proteins bind conserved elements in the abscisic acid-responsive promoter of a rice rab gene. Proc. Natl. Acad. Sci. USA 87: 1406-1410. http://dx.doi.org/10.1073/ pnas.87.4.1406

Navarro L, Dunoyer P, Jay F, Arnold B, et al. (2006). A plant miRNA contributes to antibacterial resistance by repressing auxin signaling. Science 312: 436-439. http://dx.doi.org/10.1126/science.1126088

Shen Q, Uknes SJ and Ho THD (1993). Hormone response complex in a novel abscisic acid and cycloheximide-inducible barley gene. J. Biol. Chem. 268: 23652-23660.

Shen Q, Zhang P and Ho THD (1996). Modular nature of abscisic acid (ABA) response complexes: composite promoter units that are necessary and sufficient for ABA induction of gene expression in barley. Plant Cell 8: 1107-1119. http://dx.doi.org/10.1105/tpc.8.7.1107

Sunkar R and Zhu JK (2004). Novel and stress-regulated microRNAs and other small RNAs from Arabidopsis. Plant Cell 16: 2001-2019. http://dx.doi.org/10.1105/tpc.104.022830

Várallyay E, Válóczi A, Agyi A, Burgyán J, et al. (2010). Plant virus-mediated induction of miR168 is associated with repression of ARGONAUTE1 accumulation. EMBO J. 29: 3507-3519. http://dx.doi.org/10.1038/emboj.2010.215

Vaucheret H, Mallory AC and Bartel DP (2006). AGO1 homeostasis entails coexpression of MIR168 and AGO1 and preferential stabilization of miR168 by AGO1. Mol. Cell 22: 129-136.http://dx.doi.org/10.1016/j.molcel.2006.03.011

Voinnet O (2009). Origin, biogenesis, and activity of plant microRNAs. Cell 136: 669-687. http://dx.doi.org/10.1016/j. cell.2009.01.046

Wang B, Sun YF, Song N, Wang XJ, et al. (2013). Identification of UV-B-induced microRNAs in wheat. Genet. Mol. Res. 12: 4213-4221.http://dx.doi.org/10.4238/2013.October.7.7 
Xian Z, Huang W, Yang Y, Tang N, et al. (2014). miR168 influences phase transition, leaf epinasty, and fruit development via SIAGO1s in tomato. J. Exp. Bot. 65: 6655-6666. http://dx.doi.org/10.1093/jxb/eru387

Zhou JM, Trifa Y, Silva H, Pontier D, et al. (2000). NPR1 differentially interacts with members of the TGA/OBF family of transcription factors that bind an element of the PR-1 gene required for induction by salicylic acid. Mol. Plant Microbe Interact. 13: 191-202. http://dx.doi.org/10.1094/MPMI.2000.13.2.191

\section{Supplementary material}

S1 Figure. G-box is highlighted in the MIR168 promoter of grape (two G-box) and rice (no G-box). A. MIR168 promoter sequence in rice (no G-box). B. MIR168 promoter sequence in grape (two G-boxes; highlighted). 